\title{
Non-Alcoholic Fatty Liver Disease and Associated Factors among Type 2 Diabetic Patients in Southwest Ethiopia
}

\author{
Belay Zawdie $^{1^{*}}$, Samuel Tadesse ${ }^{1}$, Amare Desalegn Wolide ${ }^{1}$, Tilahun \\ Alemayehu Nigatu ${ }^{1}$, Eshetu Mulisa Bobasa ${ }^{2}$
}

\section{OPEN ACCESS}

Citation: Belay Zawdie, Samuel Tadesse, Amare Desalegn Wolide, Tilahun Alemayehu Nigatu, Eshetu Mulisa Bobasa. Non-Alcoholic Fatty Liver Disease and Associated Factors among Type 2 Diabetic Patients in Southwest Ethiopia. Ethiop $\mathrm{J}$ Health Sci.2017;28(1):19.

doi:http://dx.doi.org/10.4314/ejhs.v28i1.4 Received: July 11, 2017

Accepted: July 14, 2017

Published: January 1, 2018

Copyright: (c) 2018 Belay Zawdie, et al . This is an open access article distributed under the terms of the Creative Commons Attribution License, which permits unrestricted use, distribution, and reproduction in any medium, provided the original author and source are credited. Funding: The research is funded by College of Public Health and Medical Sciences, Jimma University.

Competing Interests: The authors declare that this manuscript was approved by all authors in its form and that no competing interest exists.

Affiliation and Correspondence:

${ }^{1}$ School of Biomedical Sciences,

Faculty of Medical Sciences, Jimma

University

${ }^{2}$ School of Pharmacy, Faculty of

Health Sciences, Jimma University

*Email: bellzolla2000@gmail.com

\section{ABSTRACT}

BACKGROUND: Non-alcoholic Fatty Liver Disease (NAFLD) among type 2 diabetic patients is completely ignored in developing regions like Africa paving the way for public health and economic burden in the region. Therefore, the main objective of this research was to evaluate non-alcoholic fatty liver disease and associated factors among type 2 diabetic patients in Southwestern Ethiopia attending Diabetic Clinic of Jimma University Specialized Hospital (JUSH).

METHODS: Facility based cross-sectional study design was used. Anthropometry, fatty liver (using utrasonography), liver enzymes, and lipid profiles were measured among type 2 diabetic patients who fulfilled the inclusion criteria. Socio-demographic and clinical characteristics were assessed using standard questionnaires. RESULTS: Ninety-six (96) type 2 diabetic patients were enrolled and non-alcoholic fatty liver disease prevalence was 73\%. Of nonalcoholic fatty Liver disease documented patients, 35.4\%, 31.3\% and $6.3 \%$ exhibited mild, moderate and severe fatty liver diseases, respectively. Alanine aminotransferase ( $p \leq 0.001$ ), Triacyglycerol $(p \leq 0.001)$, total bilirubin $(p \leq 0.05)$, direct bilirubin $(p \leq 0.05)$ and diabetic duration $(p \leq 0.01)$ were significantly associated with nonalcoholic fatty liver disease among type 2 diabetic patients. The Aspartate aminotransferase/ Alanine aminotransferase ratio among non alcoholic fatty liver disease patients was greater than one.

CONCLUSIONS: The magnitude of non-alcoholic fatty liver disease is high among study groups and it needs urgent action by healthcare systems. Therefore, targeted treatment approach inclusive of non-alcoholic fatty liver disease should be designed.

KEYWORDS: Africa, Ethiopia, Nonalcoholic Fatty Liver Disease, Type 2 DM, Liver Enzymes, Lipid Profile

\section{INTRODUCTION}

Diabetes mellitus (DM) is one of the major public health and economic burdens among chronic non-communicable diseases worldwide (1). The cost incurred by developed nations like US in the 
management of DM is passing 100 billion dollars annually (2). It is further complicated when it is associated with Non-Alcoholic Fatty Liver Disease (NAFLD). NAFLD is "insulin resistance and hepatic fat accumulation in the absence of other identifiable causes of fat accumulation" (3, 4). The exact identification of NAFLD co-existing with DM is not yet successful making the control programs complex (5). Althouh it has remained unnoticed for many years, NAFLD is the first leading cause of liver diseases like cirrhosis $(6,7)$. Patients developing cirrhosis from NAFLD are at approximately $75 \%$ risk of acquiring liver cancer (8). The great challenge is that most of the patients do not manifest any overt signs and symptoms (8, 9), and severe NAFLD can progress to liver failure (10).

The worldwide prevalence of NAFLD is $20 \%$ in the general population and $70 \%$ amongst people with type 2 diabetes (11). More than a quarter of adults in developed nations are losing either their lives or jobs due to this disease (12). Even though there are International Diabetes Federation (IDF) reports regarding the projected prevalence of type $2 \mathrm{DM}$ to reach 1 million in Ethiopia, the number of patients developing fatty liver disease already acquiring DM is given less attention by health professionals (13).

In clinical settings, NAFLD is diagnosed through checking fatty liver along with the establishment of the nonalcoholic nature of the disease process. The best method for checking NAFLD is liver biopsy. However, ethical considerations, the high cost, its invasive nature, discomfort and risks associated with the process make it difficult to assess NAFLD in all individuals. These limitations of a liver biopsy make laboratory tests and imaging modalities indispensable methods to diagnose NAFLD in resource limited countries (3). Serum ALT and GGT are widely employed to diagnose presumed NAFLD on a population-wide basis. However, the specificity, sensitivity and predictive value of this method is under big question as evidence showed that serum ALT levels may be completely normal in patients with advanced grade of steatohepatitis or even cirrhosis. It is also studied that an elevation of ALT does not indicate the extent of hepatic damage (6). Ultrasonography, computed tomography (CT) or magnetic resonance imaging (MRI) has an adequate threshold for detection of fatty infiltration of the liver. The major limitations of these modalities include: 1) inability to differentiate steatosis from steatohepatitis and 2) insensitivity in detecting steatosis of less than 25 to $30 \%$. Of these methods, ultrasonography is the most widely available and the cheapest one. This modality has a diagnostic sensitivity of 82 to $94 \%$ and specificity greater than $82 \%$ for a fatty liver (5).

Type 2 DM and obesity were associated with NAFLD to affect the liver throughout the world (8). The findings of some researches also try to speculate the cardiovascular disease (CVD) risk of having NAFLD among type 2 diabetic patients with NAFLD as compared to type 2 diabetic patients without NAFLD. These associations could help clinicians to identify people with NAFLD who need more intensive therapy to decrease their risk of future CVD events (14-16). Many scientific data are available across the industrialized nations identifying the cause and risk factors for NAFLD among type 2 diabetic patients (6,17-19). However, the effect of NAFLD on the African population is completely ignored. Thus, it is widely feared that it may cause harsh public health and economic consequences in this part of the world (11). Therefore, the main objective of this study was to evaluate nonalcoholic fatty liver disease and associated factors among type 2 diabetic patients in Southwestern Ethiopia attending diabetic clinic of Jimma University Specialized Hospital (JUSH).

\section{METHODS AND PARTICIPANTS}

Study area and period: The study was conducted from September 2014 to February 2015 in the Diabetic Clinic of Jimma University Specialized Hospital, Jimma. Jimma University Specialized Hospital (JUSH) is the Center of Medical Excellence in Southwestern Ethiopia and probably the one with modern medical equipment in East Africa. Its establishment dates back to the first 
quarter of the $20^{\text {th }}$ century (1937 G.C.) as a military health service. It is located in Jimma Town which is about $352 \mathrm{~km}$ Southwest of the capital city, Addis Ababa, in Oromia regional state. One thousand four hundred and fifty (1450) supportive and technical staffs are hired to give services for approximately 15,000 inpatient and 160,000 outpatient attendants, 11,000 emergency cases and 4,500 deliveries in a year. Fifteen million people coming to the hospital from the catchment areas are expected to get the services of the hospital (20).

Study design: A facility based cross-sectional study design. Type 2 diabetic patients attending the Diabetic Clinic of JUSH were the study subjects. The participants who visited the Diabetic Clinic of Jimma University Specialized Hospital within the six months of the study period were thoroughly evaluated. The source for the selection was all type 2 diabetic patients' on follow-up treatment at the Diabetic Center of JUSH. The selected type 2 diabetic subjects were considered as the study population.

\section{Sample size determination and sampling} technique: Sample size was not determined initially. Rather, all type 2 diabetic patients on follow-up during the study period were randomly approached and requested for their consent to participate in the study. After their consents were obtained, the patients were evaluated for eligibility to be enrolled into the study. Nearly 1700 type 2 diabetic patients gave their consent to participate. However, based on the inclusion criteria we set for analysis of NAFLD, only 96 type 2 diabetic patients were eligible for the study.

Main procedure of the study: We excluded patients with known chronic liver disease, hepatitis B surface antigen or Anti HCV positive through diagnosis or those tested positive during the study, pregnant women, patients with history of alcohol, cigarette smokers and patients using drugs which cause fatty liver or liver toxicity using standard questioner and respective assessment of data from charts. The procedures of Gebreegziabher et al (21) and Manyazewa et al (22) with slight modifications were followed in the determination of Hepatitis B and C status with standard HBsAg test kits (Guangzhou Wondfo Biotech Co. Ltd, China). All the information was documented on a coded format prepared for this research.

Ninety-six [96] type 2 diabetic patients who fulfilled the inclusion criteria were finally included in the study. Five [5] $\mathrm{ml}$ venous blood was withdrawn from each study subject by professional nurses at the Diabetic Clinic of Jimma University Specialized Hospital. The whole blood taken was centrifuged to separate serum from plasma. The specimen was handled properly and collected in an aseptic technique and stored on ice in Biochemistry laboratory until used. On the day of clinical chemistry measurement, a formal laboratory request ordered by physicians was sent to JUSH for each selected patients. The results of each study participants were filled on clinical chemistry result report format signed by sample analyzer and approver. FPG, Liver function tests and lipid profiles were measured and analyzed by using automated machine in the Jimma University Specialized Hospital Laboratory (VegaSys automated chemistry analyzer with serial number of 201/79 Ref 16880, from AMS Via E. Barsanti 17/a, 00012 Guidonia - Rome, Italy). TG and HDL were measured according to the method described by Abda et al (23). Concentrations of Total Protein (TP), Total Bilirubin (TB) and Direct Bilirubin (DB) were determined manually (24).

Non-alcoholic fatty liver (NAFLD) checking under Ultrasound : The selected study subjects were taken under ultrasonography to evaluate the status of their liver. This activity was performed by the radiologists. The patients were given information about the type of food they should avoid (fresh fruits and vegetables, fried and oily food, milk, eggs, bread), instructed not to drink or eat at least 6 hours before the test and also not to take medications before the ultrasound. The echo of liver was compared with that of the kidney by observing through the ultrasound $(5,18,25)$.

DOI: http://dx.doi.org/10.4314/ejhs.v28i1.4 
Anthropometric measurements: All anthropometric measurements such as weight and height were made according to the WHO recommendations Guideline. Then, BMI was calculated from weight in $\mathrm{kg}$ divided by height squared $\left(\mathrm{m}^{2}\right)$ and categorized as underweight $\left(<18 \mathrm{~kg} \quad / \mathrm{m}^{2}\right)$, normal $(18-$ $\left.24.9 \mathrm{~kg} / \mathrm{m}^{2}\right)$, overweight $\left(25-29.9 \mathrm{~kg} / \mathrm{m}^{2}\right)$ and obese $\left(>30 \mathrm{kgm}^{2}\right)$ according to WHO criteria (26). The data were collected by clinical nurses selected from JUSH for assessing anthropometric measurements among selected type 2 diabetic patients in the Diabetic Ward. Data collectors were given training for three days about data collection procedures and research ethics. In addition, the process of data collection was supervised by investigators in rotation.

Data quality control: Pre-test of the tool was done on the Jimma University students who were not included in the main study by taking 5\% respondents of the total sample size to make necessary correction and completeness.

Study variables: The liver status (being without NAFL, being mild NAFL, moderate NAFL, severe NAFL), fasting blood glucose, lipid profiles and liver function tests were taken as dependent variable while independent variables were BMI, age, sex, occupation, and duration of diabetes.

Data analysis: The collected data was checked for completeness, inconsistency and outliers by looking at their distribution. Incomplete and inconsistent data were excluded from the analysis. Finally, the collected data were entered into SPSS Version 20.0. The results were put as mean \pm standard error of the mean for continuous variables and as number for categorical variables. Differences among groups of continuous variables were compared with normal (without NAFLD) type two diabetic patients using one-way ANOVA followed by Post Hoc Tukey test. Then, that of categorical variables was evaluated using chisquare. The differences were considered significant at $\mathrm{p} \leq 0.05$.
Ethical consideration: Initially, the Research and Ethical Committee of the College of Health Sciences, Jimma University, issued us approval letter. Then, formal letter was written to the Diabetic Center and the Ultrasound Unit of Jimma University Specialized Hospital. The objectives of the study were briefly explained to all the concerned bodies assigned in the hospital to get permission and support. Also, the aim of the study and its procedures were explained to the study participants who then gave written informed consent before the evaluation. The confidentiality of the data was strictly maintained throughout the study.

\section{RESULTS}

A high proportion of patients with type 2 diabetes mellitus were screened for this facility based study. However, only ninety-six [96] study participants having type 2 diabetes mellitus fulfilled the inclusion criteria. Of the sampled population, the male-to-female ratio was near to one $(46.7 \%$ males and $53.1 \%$ females). More than three fourth of the participants were older than 44 years as shown in Table 1.

As can be seen from Figure 1, more males were without NAFLD upon ultrasound liver examination than females. Nevertheless, the number of female patients with NAFLD was higher than male through all the fatty liver categories. The difference fails to attain statistically significant value despite increased number of female patients with NAFLD than males.

The majority of the normal type 2 diabetic patients were with the disease for less than two years whereas a significant number of participants with NAFLD were diagnosed with the disease before two years. The duration of diabetes for all patients with severe fatty liver disease was more than seven years (Figure 2). The clinical characteristic that significantly affected nonalcoholic fatty liver disease was duration of diabetes mellitus $(p=0.01)$. 
Non-Alcoholic Fatty Liver...

Belay Z. et al.

Table 1: Socio-demographic characteristics of sampled Type 2 Diabetic patients attending diabetic clinic of Jimma University Specialized Hospital, South West Ethiopia

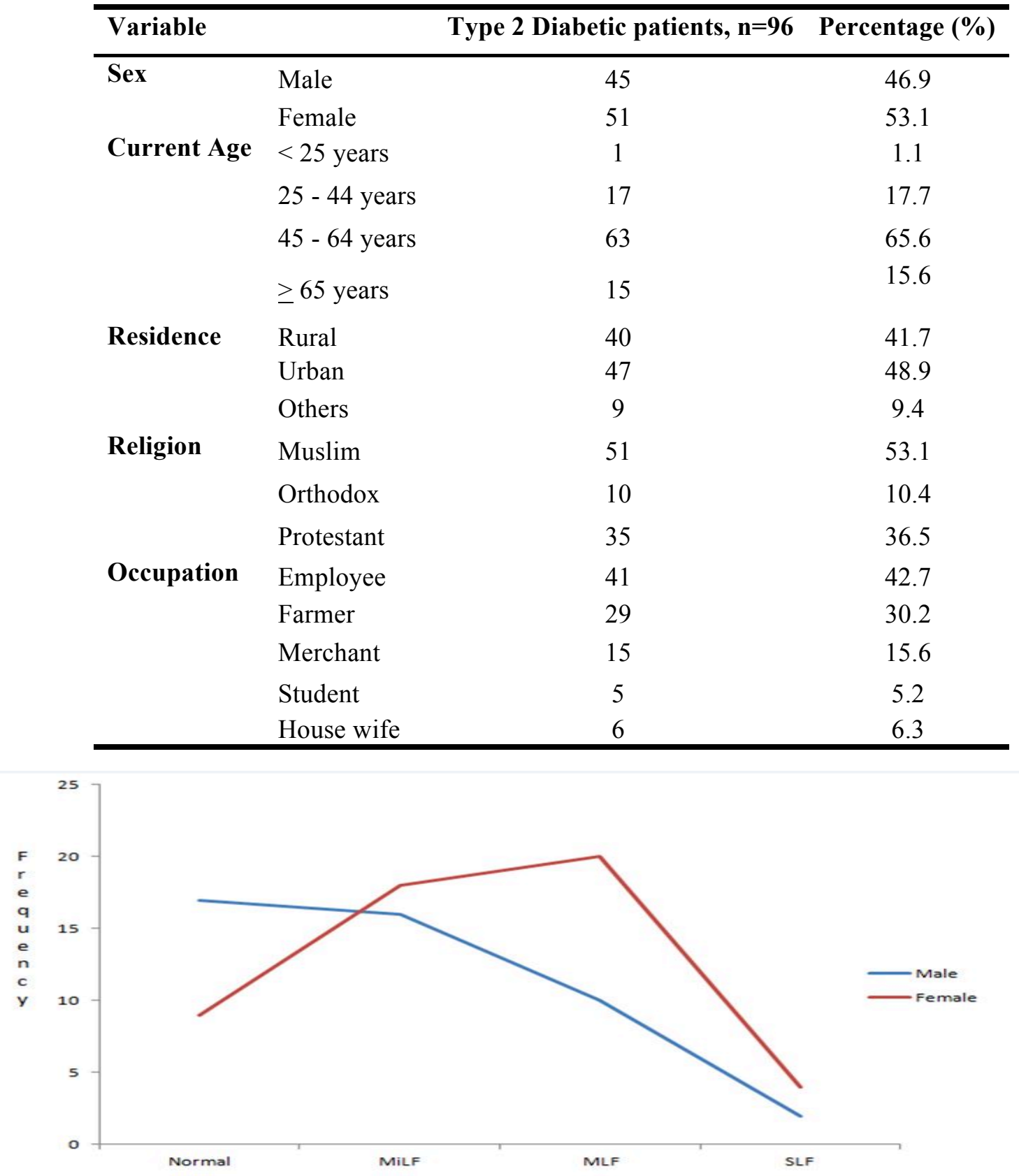

Figure 1: Liver status and sex distribution of selected type 2 diabetic patients attending diabetic clinic of JUSH, 2015. DD: diabetic duration; MiLF: Mild Fatty Liver Disease; MLF: Moderate Fatty Liver Disease; SLF: Severe Fatty Liver Disease; $p=0.101$

DOI: http://dx.doi.org/10.4314/ejhs.v28i1.4 


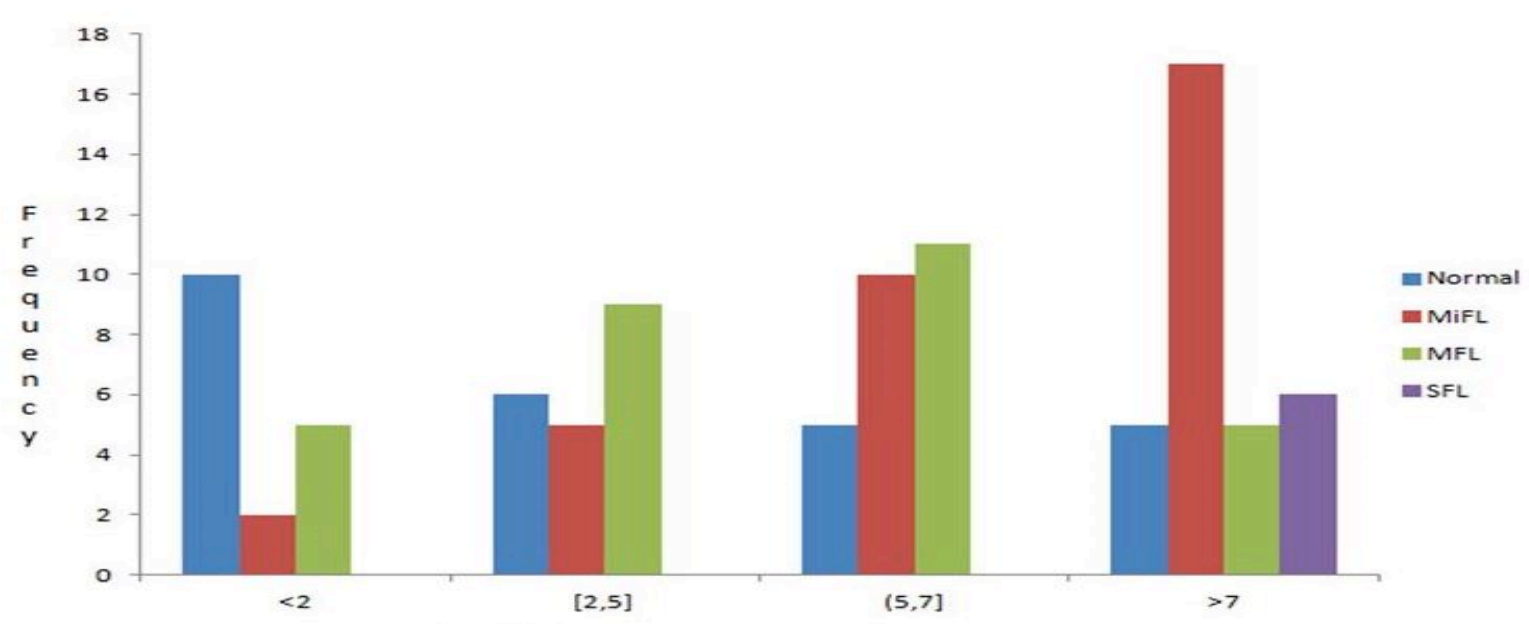

Figure 2: Diabetic duration and liver status among type 2 diabetic patients attending diabetic clinic of JUSH, 2015. DD: Diabetic Duration; MiLF: Mild Fatty Liver Disease; MLF: Moderate Fatty Liver Disease; SLF: Severe Fatty Liver Disease; DD is significantly associated at $p<0.05$

The anthropometric and clinical characteristics of the patients were presented in Table 2. Seventy of the study participants $(73 \%)$ had non-alcoholic fatty liver disease (NAFLD) whereas the remaining patients' liver ultrasound tests were negative. Of NAFLD documented patients $35.4 \%, 31.3 \%$ and $6.3 \%$ exhibited mild, moderate and severe fatty liver diseases. The calculated BMI from height and weight revealed that half of the participants had BMI in normal range (18.024.9). Twenty-eight patients had BMI in between 25 to $29.9 \mathrm{~kg} / \mathrm{m}^{2}$. The duration of diabetes mellitus for the majority of the patients was more than seven years $(34.4 \%)$, whereas $27.1 \%$ and $20.8 \%$ of them were with the disease for 5 to 7 and 2 to 5 years, respectively. One hepatitis B and three hepatitis $\mathrm{C}$ patients among participants were excluded upon screening. All the study participants did not undergo any kind of fatty liver check-up before and have any alcohol consumption history.

Table 2: Anthropometric and clinical characteristics of sampled type 2 diabetic patients attending diabetic clinic of Jimma University Specialized Hospital, Southwest Ethiopia.

\begin{tabular}{|c|c|c|}
\hline Characteristics & $\begin{array}{l}\text { Frequency, } \\
\mathrm{n}=96\end{array}$ & $\%$ \\
\hline \multicolumn{3}{|l|}{ BMI $\left(\mathrm{kg} / \mathrm{m}^{2}\right)$} \\
\hline \multicolumn{3}{|l|}{$23 \pm 0.41$} \\
\hline$<18$ & 14 & 14.6 \\
\hline $18.0-24.9$ & 50 & 52.1 \\
\hline 25.0-29.9 & 28 & 29.1 \\
\hline$>=30$ & 4 & 4.2 \\
\hline \multicolumn{3}{|l|}{$\begin{array}{l}\text { Duration of DM } \\
\text { (in Years) }\end{array}$} \\
\hline$<2$ & 17 & 17.7 \\
\hline$[2,5]$ & 20 & 20.8 \\
\hline$(5,7]$ & 26 & 27.1 \\
\hline$>7$ & 33 & 34.4 \\
\hline \multicolumn{3}{|c|}{ Liver Status Under Ultrasound } \\
\hline Normal & 26 & 27.0 \\
\hline MiLF & 34 & 35.4 \\
\hline MLF & 30 & 31.3 \\
\hline SLF & 6 & 6.3 \\
\hline \multicolumn{3}{|c|}{ Fatty Liver Checking before this study } \\
\hline Yes & 0 & 0 \\
\hline No & 96 & 100 \\
\hline \multirow{2}{*}{\multicolumn{3}{|c|}{$\begin{array}{l}\text { Alcohol } \\
\text { Consumptions }\end{array}$}} \\
\hline & & \\
\hline Yes & 0 & 0 \\
\hline No & 96 & 100 \\
\hline
\end{tabular}

DOI: http://dx.doi.org/10.4314/ejhs.v28i1.4 
The mean fasting blood glucose of the selected type 2 diabetic patients was $175 \pm 1.42(\mathrm{p}=0.67)$. Assessment of liver enzymes showed that normal type 2 diabetic patients had an averages of $11.08 \pm 0.65$ ALT while NAFL diseased patients had 15.90 \pm 1.07 among mild; $18.02 \pm 1.01$ among

Table 3: One-way ANOVA Analysis of NAFL disease with non significant biochemical and Lipid Profile tests of type 2 diabetic patients $(n=96)$

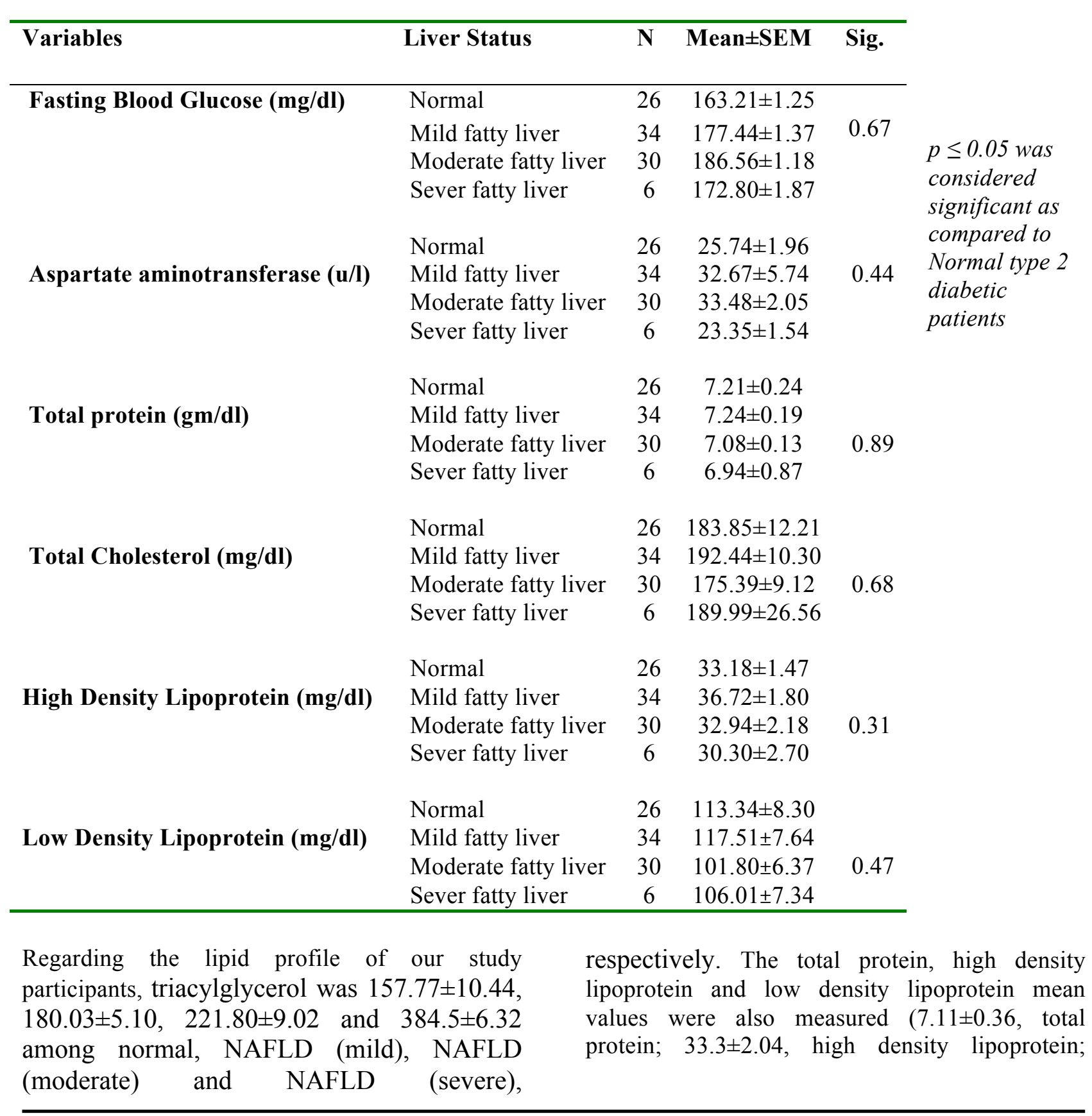

Regarding the lipid profile of our study participants, triacylglycerol was $157.77 \pm 10.44$, $180.03 \pm 5.10, \quad 221.80 \pm 9.02$ and $384.5 \pm 6.32$ among normal, NAFLD (mild), NAFLD (moderate) and NAFLD (severe), moderate; $20.05 \pm 1.27$ among severe. However, the measured values of AST and ALT were within normal reference range (Tables 3 and 4).

DOI: http://dx.doi.org/10.4314/ejhs.v28i1.4 
109.7 \pm 7.41 , low density lipoprotein (Table 3 and 4).

The statistical analysis of the study variables showed that there was a significant association between ALT, total bilirubin, direct bilirubin and triacylglycerol (TAG) concentration with nonalcoholic fatty liver diseases. ALT and TAG are strongly associated with liver status $(\mathrm{p}=0.000)$ while direct bilirubin and total bilirubin have moderate association as compared to type 2 diabetic patients without NAFLD. The mean value of ALT and direct bilirubin was significanlty different among type 2 diabetic patients with NAFD and without NAFLD at $p \leq 0.001$ and $p$ $\leq 0.05$, respectively. However, the moderate and mild fatty liver patients' mean value of total bilirubin and the mild fatty liver values of TAG were not statisticantly higher than the normal type 2 diabetic patients' laboratory result ( $p \geq 0.05$ ). Post hoc analysis revealed that the mean of ALT among severe fatty liver patients was significantly higher than the value obtained from mild fatty liver patients at $p \leq 0.05$ while the difference between moderate and mild fatty liver patients failed to attain significant difference. The comparison of TAG indicated the result among severe fatty liver patients had strong statistical difference from the mean value of moderate and mild at $p \leq 0.0001$. Unlike ALT, the mean value of TAG among moderate fatty liver patients $(221.80 \pm 9.02)$ was higher than that of mild $(180.03 \pm 5.10)(\mathrm{p}=0.001)$ (Table 4).

Table 4: One-way ANOVA and Post hoc analysis of variables significantly associated with the liver status among selected type 2 diabetic patients

\begin{tabular}{|c|c|c|c|c|}
\hline Variables & Liver Status & & Mean \pm SEM & p-value \\
\hline \multirow{4}{*}{$\operatorname{ALT}(\mathbf{u} / \mathbf{l})$} & Normal & 26 & $11.08 \pm 0.65$ & - \\
\hline & Mild Fatty Liver & 34 & $15.90 \pm 1.07$ & 0.007 \\
\hline & Moderate Fatty Liver & 30 & $18.02 \pm 1.01$ & 0.000 \\
\hline & Severe Fatty Liver & 6 & $21.72 \pm 1.37^{\mathrm{a}}$ & 0.000 \\
\hline \multirow{4}{*}{ Direct Bilirubin (gm/dl) } & Normal & 26 & $0.32 \pm 0.02$ & \\
\hline & Mild Fatty Liver & 34 & $0.23 \pm 0.02$ & 0.024 \\
\hline & Moderate Fatty Liver & 30 & $0.23 \pm 0.02$ & 0.023 \\
\hline & Severe Fatty Liver & 6 & $0.19 \pm 0.04$ & 0.012 \\
\hline \multirow{4}{*}{ Total Bilirubin (gm/dl) } & Normal & 26 & $0.54 \pm 0.04$ & \\
\hline & Mild Fatty Liver & 34 & $0.40 \pm 0.04$ & 0.118 \\
\hline & Moderate Fatty Liver & 30 & $0.46 \pm 0.05$ & 0.655 \\
\hline & Severe Fatty Liver & 6 & $0.26 \pm 0.08$ & 0.029 \\
\hline \multirow{4}{*}{ Triacylglycerol (gm/dl) } & Normal & 26 & $157.77 \pm 10.44$ & \\
\hline & Mild Fatty Liver & 34 & $180.03 \pm 5.10$ & 0.202 \\
\hline & Moderate Fatty Liver & 30 & $221.80 \pm 9.02^{\mathbf{b}}$ & 0.000 \\
\hline & Severe Fatty Liver & 6 & $384.5 \pm 6.32^{\mathrm{cd}}$ & 0.000 \\
\hline
\end{tabular}

DOI: http://dx.doi.org/10.4314/ejhs.v28i1.4 


\section{DISCUSSION}

Non-alcoholic fatty liver disease is the most serious liver disorder and cause of cirrhosis among type two diabetic patients $(7,27,28)$. Setting the objective of evaluating the occurrence of NAFLD and associated factors in type 2-Diabetic patients attending the Diabetic Center of Jimma University Specialized Hospital, Jimma, we evaluated 96 type 2 daibetic patients who fulfilled the inclusion criteria. Seventy-three percent of the study participants had NAFLD along with type 2 diabetic mellitus. The value was higher than the findings of studies done by Portillo-Sanchez et al (2015) (28), Adams et al (2010) (29), Merat et al (2009) (30) and a study done in Nigeria $(31,32)$ where the respective prevalence were $49.5 \%$, $34.4 \%, 55.8 \%$ and $16.7 \%$. The present finding is almost closer to research done in type 2 diabetic patients attending diabetic clinic of a tertiary care hospital in Mangalore (75\% prevalence) (18). This could be due to lack of liver checking habits as evidenced by the study participants' report in Table 2. The other reason might be due to low attention given by health sector on fatty liver disease. The report of NAFLD among different sexes is not conclusive. Some reports confirm as high prevalence in women while recent studies came up with even distribution (33). The gender distribution of the present study showed that more females were affected by fatty liver diseases than males (Figure 1). However, the difference was not significant at $p \leq 0.05$.

Obesity was reported as the risk factor for NAFLD. In many research findings, a fatty liver disease among type 2 diabetic patients was significantly associated with BMI $(16,18,28)$. In our findings, however, $50 \%$ of the participants had BMI in normal range (18.0-24.9) and only four patients were obese $\left(\mathrm{BMI}>30 \mathrm{~kg} / \mathrm{m}^{2}\right)$. Moreover, BMI has no significant role in the disease progression ( $\mathrm{p}>0.05)$.

Liver failure causes fats to deposit around the liver tissue resulting in fatty liver disease which may increase or decrease liver enzymes. One of the major evidences to proove the presence of NAFLD is Liver Function Tests (LFTs). It is expected that patients with NAFLD have higher liver function test abnormalities than individuals who do not have NAFLD in diabetic patients (34). It is scientifically proved that ALT is more predictive of liver fat accumulation among the liver enzymes and correlate with liver fat independent of obesity (35). The results of this study showed that ALT ( $p$ $\leq 0.001)$, total bilirubin $(p \leq 0.05)$ and direct bilirubin $(p \leq 0.05)$ are significantly associated with fatty liver diseases. Although the value of ALT is within normal range, its value is higher among NAFLD type 2 diabetic patients than normal type 2 diabetic patients. Research outputs in many other study areas showed that serum ALT levels are normal in patients with NAFLD. Hence, elevated ALT does not necessarily mean high hepatic damage (16). The AST value of NAFLD type 2 DM patients was not significantly different from that of normal type 2 patients (Table 3 ). On the other hand, the ratio of AST to ALT is greater than one in all the respective groups. The AST/ALT ratios were 2.1, 1.9 and 1.2 among mild NAFLD, moderate NAFLD and severe NAFLD type 2 diabetic patients, respectively. Patients with NAFLD usually have a ratio of AST to ALT of $<1(36)$. However, laboratory measures such as an AST to ALT ratio of $>1$ is predictors for hepatic fibrosis (35). Bilirubin has antioxidative and cytoprotectant effects (37). Report indicated inverse association of bilirubin with nonalcoholic fatty liver disease (38). The direct and total bilirubin level of the current study participants was higher among normal type 2 diabetic patients than mild and moderate NAFLD type 2 diabetic patients. Especially, type 2 diabetic patients with severe fatty liver have significantly lower bilirubin value than normal type 2 diabetic patients. Severe NAFLD patients' total bilirubin was less than normal patients by half while the record of severe fatty liver patients' direct bilirun was less by slightly higher than $40 \%$ (Tables 3 and 4 ).

DOI: http://dx.doi.org/10.4314/ejhs.v28i1.4 
Triacylglycerol (TAG) is one of the main factors affecting NAFLD in the present study. The mean value of TAG among type 2 diabetic patients with severe fatty liver was two times the laboratory mean results of normal and mild fatty liver patients. NAFLD is highly bonded with TAG accumulation in the hepatocytes. This store may arise from different sources including the intestine (through absorption), and the liver (synthesis). The high level of glucose or insulin will activate some transcription factors resulting in increased hepatic de novo lipogenesis. Finally, excessive lipolysis will form steatosis $(4,6,12,39)$. Thus, the high level of TAG may be due to hyperglycemia and hyperinsulinemia as evidenced by the poor glycemic control (mean of FPG 175 \pm 1.42 ) (Table $3)$.

In conclusion the prevalence of NAFLD at the study site was $73 \%$. ALT, TAG, direct and total bilirubin were the major significant determinants of NAFLD occurrence among type 2 diabetic patients by either affecting the disease progression in positive or negative linear proportion. Type 2 diabetic patients with NAFLD have significantly longer diabetic duration than normal type 2 diabetic patients. Even though AST is not significantly associated with NAFLD; the $\mathrm{AST} / \mathrm{ALT}$ ratio is greater than one.

Non-alcoholic fatty liver diseases are the major risk factors for developing cardiovascular diseases (16), stroke, peripheral vascular disease (11), chronic kidney disease (40), cirrhosis and liver cancer (6) among type 2 diabetic patients. Therefore, the authors recommend further research across Ethiopia to know the pathogenesis and identify effective treatment options inclusive of NAFLD.

While there is a real need to understand the true impact of NAFLD in developing countries and especially among type $2 \mathrm{DM}$ patients, the size of the study population may not add much information to our current knowledge about the epidemiology of NAFLD in Ethiopia. Beside, the use of ultrasound and serum enzymes as the sole marker of NAFLD is another limitation as both modalities are not the most sensitive, specific and predictive method for detecting (quantifying) liver fat despite being cheap and accessible in developing countries like Ethiopia.

\section{ACKNOWLEDGEMENTS}

The authors would like to acknowledge Dr Elias Kedir (MD, Radiologist) for his time and cooperation in the diagnosis of the patients. And our gratitude also goes to all data collectors who work hard for this research. We acknowledge again College of Public Health and Medical Sciences, Jimma University, for funding this research.

\section{REFERENCES}

1. Uttra KM, Devrajani BR, Shah SZA, Devrajani T, Das T, Raza S, Naseem. Lipid Profile of Patients with Diabetes Mellitus. World Appl Sci J 2011; 12 (9): 1382-84.

2. Couric H, Boyko R, Bennet S. Diabetes in America, 2nd ed. Washington DC: U.S. Printing Office; NIH publication 1995; 951468.

3. Chalasani N, Younossi Z, Lavine JE, Diehl AM, Brunt EM, Cusi K, Charlton M, Sanyal AJ. The Diagnosis and Management of NonAlcoholic Fatty Liver Disease: Practice Guideline by the American Association for the Study of Liver Diseases, American College of Gastroenterology, and the American Gastroenterological Association. Hepatol 2012; 55 (6): 2005-22.

4. Cusi K. Nonalcoholic fatty liver disease in type 2 diabetes mellitus. Curr Opin Endocrinol Diabetes Obes 2009; 16:141-9.

5. Shannon A, Alkhouri N, Carter-Kent C, Monti L, Devito R, Lopez R, Feldstein AE, Nobili V. Ultrasonographic Quantitative Estimation of Hepatic Steatosis in Children with Nonalcoholic Fatty Liver Disease (NAFLD). $J$ Pediatr Gastroenterol Nutr 2011; 53(2): 19095.

6. Hazlehurst JM, Woods C, Marjot T, Cobbold $\mathrm{JF}$, Tomlinsona JW. Non-alcoholic fatty liver disease and diabetes. Metabolism 2016; 65 (8): 1096-108.

DOI: http://dx.doi.org/10.4314/ejhs.v28i1.4 
7. Vanni B, Marchesini K. NASH and the risk of cirrhosis and Hepatocellular carcinoma in type 2 diabetes. Curr Diabetes Rep 2007; 7:17580 .

8. Bhatt HB, Smith RJ. Fatty liver disease in diabetes mellitus. Hepato Biliary Surg Nutr 2015; 4(2):101-108.

9. Collantes R, Ong JP, Younossi ZM. Nonalcoholic fatty liver disease and the epidemic of obesity. Cleve Clin J Med 2004; 71: 657-64.

10. Shaib DM, El-Serag M. Diabetes increases the risk of hepatocellular carcinoma in the United States: a population basal case Control study. Gut 2005; 54: 533-39.

11. Wainwright P. Non-alcoholic fatty liver disease and type 2 diabetes: An overview of the problem. J Diabetes Nurs 2015; 19: 19599.

12. Firneisz G. Non-alcoholic fatty liver disease and type 2 diabetes mellitus: The liver disease of our age. World J Gastroenterol 2014; 20(27): 9072-9089.

13. International Diabetic Federation annual report 2011. Accessed on September, 2016 at https://www.idf.org/sites/default/files/attachm ents/IDF-AR2013-final-rv.pdf

14. Saksena D. Non-alcoholic steatohepatitis: definitions and pathogenesis. $J$ Gastroenterol Hepatol 2002; 17 (Suppl 3): S377-S384.

15. Utzschneider K, Steven J. The Role of Insulin Resistance in Nonalcoholic Fatty Liver Disease. J Clin Endocrinol Metab2004; 91(12):4753-476.

16. Targher G, Bertolini L, Padovani R, Rodella S, Tessari R, Zenari L, Day C, Arcaro G. Prevalence of Nonalcoholic Fatty Liver Disease and Its Association with Cardiovascular Disease among Type 2 Diabetic Patients. Diabetes Care 2007; 30 (5): 1212-18.

17. Jia G, Di F, Wang Q, Shao J, Gao L, Wang L, et al. Non-Alcoholic Fatty Liver Disease Is a Risk Factor for the Development of Diabetic Nephropathy in Patients with Type 2 Diabetes Mellitus. PLoS ONE 2015; 10(11): e0142808.

18. ShivanandaPai M, chakrapani M, Deepak R Madi, Basavaprabhu Achappa, Unnikrishnan.
Non alcoholic fatty liver disease in patients with type two diabetes mellitus. IJBMR 2012; 3 (3): 2189-92.

19. Zhang $\mathrm{J}$ et al. Association between serum free fatty acid levels and nonalcoholic fatty liver disease: a cross-sectional study. Sci Rep 2014; 4: 5832.

20. Accessed on May, 2014 at https://www.ju.edu.et/jimma-universityspecialized-hospital-jush,

21. Gebreegziabher D, Asfeha GG, Gebreyesus HA. Seroprevalence of hepatitis B virus surface antigen (HBsAg) among clients visiting 'Tefera Hailu' memorial hospital, Sekota, Northern Ethiopia. BMC Infect Dis 2016; 16:383.

22. Manyazewa T, Sisay Z, Biadgilign S, Abegaz WE. Hepatitis B and hepatitis C virus infections among antiretroviral-naive and experienced HIV co-infected adults. $J$ Med Microbiol 2014; 63:742-47.

23. Abda E, Hamza L, Tessema F, Cheneke W. Metabolic syndrome and associated factors among outpatients of Jimma University Teaching Hospital. Diabetes Metab Syndr Obes: Targets and Therapy 2016:9 47-53.

24. Esteghamati et al. Metabolic syndrome is linked to a mild elevation in liver aminotransferases in diabetic patients with undetectable non-alcoholic fatty liver disease by ultrasound. Diabetol Metab Syndr 2010; 2:65.

25. Nagaraj S, Kiran SS, Gandham R, Silvia WDCR, Nagaraja MR, Nasar AS, et al. Study of prevalence of non alcoholic fatty liver disease in type 2 diabetes mellitus patients and variations in liver function tests, lipid profile and mean platelet volume in patients with fatty liver in comparison with patients without fatty liver. Int J Res Med Sci 2016; 4:871-6.

26. World Health Organization. WHO child growth standards: length/height-for-age, weight-for-length, weight for height, and body mass index for age: methods \& development. Geneva. World Health Organization, 2006.

27. Salt WB. Nonalcoholic Fatty Liver Disease (NAFLD): A Comprehensive Review. Insur Med 2004; 36:27-41. 
28. Portillo-Sanchez P et al. High Prevalence of Nonalcoholic Fatty Liver Disease in Patients with Type 2 Diabetes Mellitus and Normal Plasma Aminotransferase Levels. $J$ Clin Endocrinol Metab 2015; 100(6):2231-38.

29. Adams LA, Harmsen S, Sauver JLS, Charatcharoenwitthaya P, Enders FB, Therneau T, Angulo P. Nonalcoholic Fatty Liver Disease Increases Risk of Death Among Patients With Diabetes: A Community-Based Cohort Study. Am J Gastroenterol 2010; 105:1567-1573.

30. Merat S, Yarahmadi S, Tahaghoghi S, Alizadeh Z, Sedighi N, Mansournia N, Ghorbani A, Malekzadeh R. Prevalence of Fatty Liver Disease among Type 2 Diabetes Mellitus Patients and its Relation to Insulin Resistance. Middle East J Dig Dis 2009; 1(2): 74-9.

31. Onyekwere CA, Ogbega AO, Balogun BO. Non-Alcoholic Fatty Liver Disease and the metabolic syndrome in an urban hospital serving an Africa community. Ann hepatol 2011; 10(2):119-24.

32. Olusanya TO, Lesi OA, Adeyomoye AA, Fasanmade OA. Non alcoholic fatty liver disease in a Nigerian population with type II diabetes mellitus. Pan Afr Med J 2016; 24:20.

33. Lazo M, Clark JM. The Epidemiology of Nonalcoholic Fatty Liver Disease: A Global
Perspective: Sociodemographic Differences in the Prevalence of NAFLD. Semin Liver Dis 2008; 28(4):339-350.

34. Harris EH. Elevated Liver Function Tests in Type 2 Diabetes. Clin Diabetes 2005; 23: 115.

35. Neely D. Liver enzymes, fatty liver and type 2 diabetes. Ann Clin Biochem 2005; 42: 167169.

36. Angulo P. Obesity and nonalcoholic fatty liver disease. Nutr Rev 2007; 65:S57- 63.

37. Tian, J. et al. Association between bilirubin and risk of Non-Alcoholic Fatty Liver Disease based on a prospective cohort study. Sci Rep 2016; 6: 310-06.

38. Kwak M, et al. Serum bilirubin levels are inversely associated with nonalcoholic fatty liver disease. Clin Mol Hepatol 2012; 18 (4): 383-90.

39. Smith D, Jessurun S, Parks B. Sources of fatty acids stored in liver and secreted via lipoproteins in patients with nonalcoholic fatty liver disease. J Clin Invest 2005; 115:13431351.

40. Targher G, Choncho M, Bertolini L, Rodella S, Zenari L, Lippi G, Franchini M, Zoppini G, Muggeo M. Increased Risk of CKD among Type 2 Diabetics with Nonalcoholic Fatty Liver Disease. J Am Soc Nephrol 2008; 19: 1564-1570. 